\title{
Adhesive strength of metal brackets on existing composite, amalgam and restoration-enamel complex following air-abrasion protocols
}

Yetkiner, Enver ; Özcan, Mutlu

DOI: https://doi.org/10.1016/j.ijadhadh.2014.06.012

Posted at the Zurich Open Repository and Archive, University of Zurich ZORA URL: https://doi.org/10.5167/uzh-100659

Journal Article

Accepted Version

Originally published at:

Yetkiner, Enver; Ȯzcan, Mutlu (2014). Adhesive strength of metal brackets on existing composite, amalgam and restoration-enamel complex following air-abrasion protocols. International Journal of Adhesion and Adhesives, 54:200-205.

DOI: https://doi.org/10.1016/j.ijadhadh.2014.06.012 
Adhesive strength of metal brackets on existing composite, amalgam and restoration-enamel complex following air-abrasion protocols

Enver Yetkiner ${ }^{a,}$, Mutlu Özcan $^{\mathrm{b}}$

${ }^{a}$ Department of Orthodontics, University of Ege, Izmir, Turkey

${ }^{b}$ Dental Materials Unit, University of Zürich, Center for Dental and Oral Medicine, Clinic for Fixed and Removable Prosthodontics and Dental Materials Science, Plattenstrasse 11, CH-8032 Zürich, Switzerland

Short Title: Adhesion of brackets on restoration materials using air-abrasion and silanes

Part of this study has been presented at the 13th Symposium of Turkish Ortodontic Society, November, 3rd-5th, 2013, Istanbul, Turkey.

${ }^{*}$ Correspondance to: Dr. Enver Yetkiner, University of Ege, Department of Orthodontics, 35100, Izmir, Turkey. Tel: +90-232-311 4592, Fax: +90-232-388 0325. E-mail: eyetkiner@hotmail.com (E. Yetkiner) 


\section{Abstract}

Bracket adhesion on restored tooth surfaces is occasionally necessary in clinical orthodontic practice. The objective of this study was to compare the effects of two air-abrasion methods on adhesion of metal brackets to enamel, resin composite, amalgam and composite/amalgam-enamel complexes. Cavities in standard dimensions $\left(12.56 \mathrm{~mm}^{2}\right.$ ) were filled with resin composite (Anterior Shine, Cavex) and amalgam (Non-gamma 2, Cavex) on bovine incisors $(\mathrm{N}=40)$, which were then embedded in acrylic resin. Metal brackets were bonded on the following surfaces ( $n=10$ per group): 1) Enamel, 2) Enamel-Composite, 3) Enamel-Amalgam, 4) Composite, and 5) Amalgam. All restorative materials were either silica-coated with

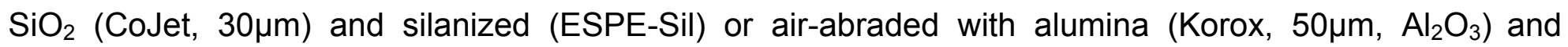
silanized (Monobond Plus). Enamel was etched with $\mathrm{H}_{3} \mathrm{PO}_{4}$ for $30 \mathrm{~s}$ in Groups 1, 2 and 3. Metal brackets were bonded onto the conditioned substrates. Specimens were stored in distilled water $\left(24 \mathrm{~h}, 37^{\circ} \mathrm{C}\right)$ following bonding. Brackets were then debonded using Universal Testing Machine $(1 \mathrm{~mm} / \mathrm{min})$. Shear bond strength (SBS) data were recorded and failure types were categorized. Data (MPa) were analyzed using 1way and 2-way ANOVA, Tukey's post-hoc test and 2-parameter Weibull distribution. While substrate type significantly affected the SBS $(p<0.001)$, surface conditioning did not show a significant effect $(p=0.256)$. Interaction terms were not significant $(p=0.159)$. Mean SBS was significantly higher $(p<0.001)$ on enamel (26.72 MPa), composite (29.97-31.37 MPa) and enamel+silica-coated composite complex (25.89 MPa) than those of other groups (10.96-20.64 MPa). Presence of amalgam resulted in the lowest SBS regardless of the conditioning method (10.96-12.41). Air-abrasion with $\mathrm{Al}_{2} \mathrm{O}_{3}$ followed by Monobond Plus and silica-coating and silanization did not show significant difference $(p>0.05)$. Weibull distribution presented lower shape for restoration-enamel complexes (2.20-6.31) compared to single component surfaces (10.14-12.15). SBS on composite was similar to enamel but presented predominantly cohesive failures. Failure types were frequently cohesive in composite alone or composite-enamel complex.

Keywords: Adhesion, Amalgam, Enamel, Orthodontics, Primers, Silane coupling agents, Surface conditioning 


\section{Introduction}

Bracket adhesion on sound enamel depends primarily on resin tag formation within the etched surface, providing micro-mechanical retention. In clinical practice however, bonding brackets on compromised tooth surfaces might be necessary when restorations are present in the targeted bonding area [1]. Resin composite, amalgam, ceramic and gold are the commonly encountered restorative materials [2]. Especially with the increase in adult patients, orthodontists are more likely to bond brackets onto composite and amalgam restorations on the buccal tooth surfaces depending on the location. A vast number of studies have been performed investigating the adhesive performance of brackets on restorative material surfaces [3-9]. Additional surface conditioning methods increasing surface roughness and the use of intermediate adhesive resin have been reported to improve bond strength on such surfaces [3-8]. These procedures have become a part of the routine clinical practice, aiming surface area increase for better micromechanical retention and at the same time forming chemical bonds between the adhesive and the restorative materials $[9,10]$.

Increasing the surface area can be achieved by either abrading the surface with burs [6,7] or by airborne particle abrasion (hereon: air-abrasion) with $\mathrm{Al}_{2} \underline{O}_{3}$ or $\mathrm{SiO}_{2}$ [11]. Air-abrasion produces etchedenamel like surfaces with a significant surface area increase [8,10-12] where air-abrasion with $\mathrm{SiO}_{2}$, the so called silica-coating, has presented the additional advantage of providing a chemically active surface, which was then enhanced by the application of silane coupling agents. Commercial silanes contain chemical adhesion promoters such as silane methacrylate, phosphoric acid methacrylate and sulfide methacrylate through which adhesion could be enhanced [10,[11]. This improvement is due to covalent bonds formed between the adhesive resin and the coated area, which is considered adjunct to the mechanical retention increasing bond strength of resin-based materials to different substrates $[8,10-12,13]$.

Recently, a new silane-coupling agent, universal primer, has been introduced for conditioning all types of restoration materials which is a combination of the above mentioned adhesion promoters [14]. Alternative to the commonly used silane, 3-methacryloxyproyltrimethoxysilane (MPS), these new primers contain 
cyclic disulphide, also enhancing adhesion to precious alloys. Adhesion between ceramic and luting composites using this new primer has been investigated previously [14]. However, there is no data reported regarding the bracket adhesion on composite or amalgam using this silane after surface conditioning methods based on air-abrasion protocols.

The uniformity of the targeted bonding area in orthodontics is another factor influencing the performance of contemporary adhesive procedures since at least two interfaces are of consideration: substrate surface -adhesive resin interface and adhesive resin-bracket base interface [9]. The different physical and chemical properties of these components determine the conditions of adhesion in orthodontics [9]. When the bonding area consists of not only restorative material but also the neighbouring enamel, then three substances with different physical and chemical properties are subject to surface conditioning.

The objective of this study therefore was to evaluate the bond strength of metal brackets on amalgam or composite restorative materials and on amalgam-enamel, composite-enamel complexes following two surface conditioning procedures. The tested hypotheses were that air-abrasion with $\mathrm{Al}_{2} \mathrm{O}_{3}$ followed by universal primer would provide similar bond strength compared to silica-coating and MPS silane coupling application and that bonding brackets on restoration margins would present lower bond strength than to restoration material or enamel alone.

\section{Experimental}

\subsection{Materials and Methods}

The brands, types, abbreviations, chemical compositions and manufacturers of the materials used for the experiments are listed in Table 1.

\subsubsection{Specimen preparation}

Coronal parts of bovine mandibular incisors $(\mathrm{N}=40)$ stored in $0.5 \%$ chloramine solution at $4^{\circ} \mathrm{C}$ no longer than 6 months were initially cut from their roots using a low-speed diamond bur (Isomet, Buehler, Illinois, USA) under constant water-cooling. They were embedded with their labial surfaces exposed in auto- 
polymerized acrylic resin (Palapress, Vario, Hereaus Kulzer, Wehrheim, Germany) in cylindrical moulds (diameter: $25 \mathrm{~mm}$; UnoForm, Struers, Bellerup, Denmark). Specimens were then ground flat and polished with water-cooled carborundum discs (1200, 2400 and 4000 grit, Struers, Erkrat, Germany). Cavities of standard size $\left(12.56 \mathrm{~mm}^{2}\right)$ on mesial and distal aspects of each crown were prepared using a custommade diamond-coated trephine (inner $\varnothing=2 \mathrm{~mm}, 80 \mu \mathrm{m}$ ) (Intensiv SA, Lugano-Grancia, Switzerland) under water cooling. One of the two cavities on each specimen was etched with $37 \% \mathrm{H}_{3} \mathrm{PO}_{4}$ (Orbis Dental, Munster, Germany) for $30 \mathrm{~s}$, rinsed with water spray for $30 \mathrm{~s}$ and dried with compressed oil-free air. A coat of primer was applied for $15 \mathrm{~s}$ and gently air-thinned for $5 \mathrm{~s}$. Then, a coat of bonding agent (Quadrant Unibond Sealer, Cavex, Haarlem, The Netherlands) was applied, air-thinned and photo-polymerized for 20 s. Resin composite (Anterior Shine, Cavex Holland BV) was applied in three increments forming a smooth surface and photo-polymerized using an LED polymerization device for $40 \mathrm{~s}$ (Epilar Freelight II LED, 3M ESPE, Seefeld, Germany; Output $=1000 \mathrm{~mW} / \mathrm{cm}^{2}$ ) from a distance of $2 \mathrm{~mm}$. Amalgam (Lathe-cut, NonGamma 2, Cavex Holland BV, Haarlem, The Netherlands) was condensed in the remaining cavities of the specimens until forming a smooth surface and polished with a burnisher. All specimens were re-polished with water-cooled carborundum discs (2400 and 4000 grit, Struers) in order to standardize the bonding surface for optimum bracket base adaptation. The specimens were stored in distilled water for another 48 hours at $37^{\circ} \mathrm{C}$ and randomly assigned to two groups for surface conditioning.

\subsubsection{Surface conditioning}

Silica coating and silanization: Amalgam and composite surfaces were silica-coated ( $30 \mu \mathrm{m} \mathrm{Al}_{2} \mathrm{O}_{3}$ particles modified by silica, CoJet Sand, 3M ESPE, Seefeld, Germany) using an intraoral air-abrasion device (Microetcher, Danville Eng., San Ramon, CA, USA) with a nozzle distance of approximately $10 \mathrm{~mm}$ at a vertical angle for $4 \mathrm{~s}$ at $3 \mathrm{psi}$. Then, MPS silane (ESPE-Sil, 3M ESPE) was applied every time with a new microbrush and waited for its reaction for $30 \mathrm{~s}$.

Air-abrasion with $\mathrm{Al}_{2} \mathrm{O}_{3}$ and silanization: Amalgam and composite surfaces were air-abraded (50 $\mu \mathrm{m} \mathrm{Al}_{2} \mathrm{O}_{3}$ particles, Korox Sand, Bego, Bremen, Germany) with the same parameters used for silica coating. Then, 
the silane (Monobond Plus, Ivoclar Vivadent, Schaan, Liechtenstein) was applied every time with a microbrush and waited for its reaction for $60 \mathrm{~s}$.

\subsubsection{Bracket bonding}

Following these pre-treatments, metal brackets with $8.71 \mathrm{~mm}^{2}$ laser-structured bases for central lower incisors (Discovery, slot $0.56 \cdot 0.76 \mathrm{~mm} / 22 \cdot 30$, Dentaurum, Ispringen, Germany) were bonded on specimens using a photo-polymerized conventional primer and adhesive paste (Transbond XT, 3M Unitek, Monrovia, USA) under a standard load of $500 \mathrm{~g}$. Excess resin was removed using foam pellets. Photopolymerization was achieved using LED polymerization device (Epilar Freelight II LED, 3M ESPE, Seefeld,

Germany; Output $=1000 \mathrm{~mW} / \mathrm{cm}^{2}$ ) for $15 \mathrm{~s}$ from incisal, gingival, mesial and distal directions. The vertical line on brackets guiding the vertical axis was kept parallel to the medial side of restorations for standard positioning of the brackets. The first group of 10 specimens received 30 brackets bonded on amalgam$\mathrm{Al}_{2} \mathrm{O}_{3}$, composite- $\mathrm{Al}_{2} \mathrm{O}_{3}$ and etched enamel. The second group of 10 specimens received 20 brackets bonded on silica coated amalgam and silica coated composite. The third group of 10 specimens received 20 brackets bonded on etched enamel-air-abraded amalgam and enamel- air-abraded composite. Finally, The fourth group of 10 specimens received 20 brackets bonded on etched enamel-silica coated amalgam and enamel-silica coated composite. In summary, there were 40 teeth, 90 brackets in total and 10 brackets per group. The complex surfaces were bonded on the same specimen to keep the enamel component stable. Flowchart of the experimental sequence, position of the amalgam and composite restorations and the bonded brackets on bovine teeth are illustrated in Figs. 1 and 2.

\subsubsection{Shear bond strength test (SBS)}

Brackets were debonded from substrate surfaces using SBS test in a Universal Testing Machine (Z010, Zwick, Ulm, Germany). A stainless steel rod with a chisel configuration was used for debonding (crosshead speed: $1 \mathrm{~mm} / \mathrm{min}$ ). Load at failure was recorded and bond strength values were calculated according to the following equation: $\mathrm{S}=\mathrm{F} / \mathrm{A}$, where $\mathrm{S}$ is the bond strength $(\mathrm{MPa}), \mathrm{F}$ is load at failure $(\mathrm{N})$, and $\mathrm{A}$ the adhesive area $\left(\mathrm{mm}^{2}\right)$. 


\subsubsection{Failure mode analysis}

Following SBS, substrate surfaces of all specimens were inspected under optical stereomicroscope (Zeiss, Göttingen, Germany) at x10 magnification. Failure modes were classified as follows: a) adhesive: when failure was between bracket and substrate with no remnants of resin on the substrate surface, b) cohesive: when the substrate failed with damaged integrity and c) mixed: when a combination of the adhesive failure from substrate and cohesive failure of the substrate or adhesive resin was present.

\subsection{Statistical analysis}

A sample size of 10 in each group was calculated to have more than $80 \%$ power to detect a difference of 7.45 MPa between mean values. According to the two-group Satterthwaite t-test (SPSS Software V.20, Chicago, IL, USA) with a 0.05 two-sided significance level, this assumes that for conditjoning wirh $\mathrm{Al}_{2} \mathrm{O}_{3}$ standard deviation is 5.38 and with $\mathrm{SiO}_{2}$ standard deviation is 5.02. Kolmogorov-Smirnov and Shapiro-Wilk tests were used to test normal distribution of the data. As the data were normally distributed, 1-way and 2way analysis of variance (ANOVA) were applied to analyse possible differences between the groups. Interaction of substrate surfaces and surface conditioning methods were analyzed using Tukey's post-hoc test. Maximum likelihood estimation without a correction factor was used for 2-parameter Weibull distribution to interpret predictability and reliability of adhesion (Minitab Software V.16, State College, PA, USA). Level for significance was set at $p<0.05$ for all tests.

\section{Results}

While substrate type significantly affected the SBS $(p<0.001)$, surface conditioning did not show a significant effect $(p=0.256)$. Interaction terms were not significant $(p=0.159)$.

Mean SBS was significantly higher $(p<0.001)$ on enamel $(26.72 \mathrm{MPa})$, composite $(29.97-31.37 \mathrm{MPa})$ and enamel-silica coated composite complex $(25.89 \mathrm{MPa})$ than those of the other groups (10.96-20.64 MPa). Presence of amalgam resulted in the lowest SBS regardless of the conditioning method (10.9612.41). Air-abrasion with $\mathrm{Al}_{2} \mathrm{O}_{3}$ followed by Monobond Plus and silica-coating and silanization did not show significant difference $(p>0.05)$. 
Weibull distribution presented high scale $(\sigma)$ values and good fit with Weibull curves for enamel, composite and amalgam surfaces indicating high reliability of the adhesion. Bonding on complex surfaces regardless of surface conditioning methods created a distortion in the Weibull fit with significantly lower shape values. Weibull distribution presented lower shape for restoration-enamel complexes (2.20-6.31) compared to single component surfaces (10.14-12.15). Scale and shape values together with the Weibull probability plot for all groups with 95\% confidence intervals are displayed in Fig. 3.

Specimens involving amalgam and amalgam-enamel presented predominantly adhesive failure type $(80-100 \%)$ (Table 3). Enamel alone and composite-enamel specimens presented mostly mixed failures $(60-80 \%)$ whereas composite specimens, regardless of the surface conditioning method, revealed frequently cohesive failures $(70 \%)$.

\section{Discussion}

This study investigated the adhesion of metal brackets on enamel, composite, amalgam and enamelcomposite and enamel-amalgam complexes following two surface conditioning methods. Since airabrasion with $\mathrm{Al}_{2} \mathrm{O}_{3}$ followed by universal primer application provided similar shear bond strength with silica-coating and silanization, the first hypothesis was accepted. This also implies that the new silane could substitute silica-coating and MPS silanization for amalgam and composite surfaces.

Bonding brackets to complex surfaces decreased the bond strength for enamel-amalgam substrates but not for composite-enamel. Therefore, the second hypothesis is partly rejected. This may be attributed to the contamination of amalgam surface during etching of neighbouring enamel, resulting in a possible interference for the silane mechanism together with weaker bonds formed between two physically and chemically different materials. Consequently, contamination of the restoration surface with phosphoric acid during the etching of neighbouring enamel might adversely affect the chemical reaction with the silane. Thus, it can be stated that presence of complex bonding surfaces might have an adverse effect on the adhesion of brackets bonded on restoration margins. 
Bovine teeth were chosen in this study in order to facilitate experiments on three different substrates and their combinations in one specimen. Although it has been shown that bond strength measurements to bovine enamel are slightly inferior compared to human enamel, it has been suggested that bovine enamel could be safely used, particularly when a large crown size is needed [16].

Clinically adequate bond strength for metal brackets to enamel has been recommended as 6-8 MPa [17] even though the use of this reference value has been subjected to criticism [18]. A number of factors influence the outcome of bond tests such as substrate surface properties, surface topography, bonding area, application mode of bracket placement and administration of shear force in terms of location and direction [18]. In the present study, brackets bonded to enamel and different restoration materials revealed higher mean shear bond strengths $(10.96-31.37 \mathrm{MPa})$ when compared to the reference range. This is usually the case for in vitro test results due to improved bonding conditions such as isolation of moisture, flat bracket base-enamel surface adaptation, constant application of force during excess resin removal and photo-polymerization. Despite the fact that the actual debonding mechanism of orthodontic attachments is not caused by pure shear force, this testing method is helpful in examining performance of various materials. Therefore, the resultant data could be used for ranking products and protocols within a single study $[9,18]$.

Previously, bond strength of luting composite to restorative composite surfaces was shown to be successful regardless of the surface conditioning methods [4,7,19]. Accordingly, in the present study composite specimens presented significantly higher bond strength compared to amalgam and enamelamalgam groups irrespective of the surface conditioning method. Bonding to enamel-amalgam complex performed better than amalgam alone, yet this difference was not significant. Similarly, earlier studies revealed inferior bond strength on surface conditioned amalgam $[3,8]$, but no data was present on complex surfaces such as enamel-amalgam and enamel-composite combinations. Presence of enamel adjacent to amalgam in the bonding area increased the bond strength although this was not the case for composite specimens. Composite to composite adhesion provided the highest shear bond strength for both 
conditioning methods but this connection was adversely affected when enamel was involved. Interestingly, Weibull probability plot revealed low predictability for all complex surface specimens irrespective of conditioning methods. In particular, silica coated enamel-composite complex and enamel- $\mathrm{Al}_{2} \mathrm{O}$ air abraded amalgam complex presented less steep slopes resulting in lower Weibull modulus and characteristic life. Therefore, this distortion can be interpreted as a possible indicator of complex surfaces being unpredictable for bracket bonding although it may increase bond strength as in the example of enamelamalgam specimens [20]. These assumptions need to be verified in clinical studies.

In this study, amalgam and enamel-amalgam specimens exhibited mostly adhesive failures, whereas composite specimens presented generally cohesive failures. This implies that amalgam-adhesive resin interface exhibited lower bond strength compared to the cohesive strength of the adhesive resin itself. Likewise, composite-adhesive resin interface provided higher bond strength than the cohesive strength of the restorative composite, which resulted in chipping of the restoration following debonding. Unlike restorative dentistry, bonding in orthodontics does not require permanent adhesion but rather resilient bonding during the whole course of treatment. At the end of the treatment, debonding should not damage the substrate surface $[7,8,19]$. Otherwise, some repair actions need to be undertaken. The high incidence of cohesive failures obtained in the composite group requires questioning the necessity of such a surface conditioning method prior to orthodontic bonding.

The non-significant difference between the two air-abrasion protocols could be attributed to the silanecoupling agents used in these systems. While silica-coating system requires the use of MPS silane subsequent to air-abrasion with silica, according to the manufacturer's instructions of the universal primer, Monobond Plus, air-abrasion with alumina is sufficient. Universal primer having phosphoric acid ester and MPS silane in its composition could co-polymerize with oxide and hydroxyl groups of a given substrate after alumina air-abrasion [20]. Yet, the stability of the adhesion needs to be verified after long-term hydrothermal aging. 


\section{Conclusions}

From this study, the following could be concluded:

1. Shear bond strength of metal brackets to air-abraded and silanized amalgam was lower when compared to composite or etched enamel alone.

2. Conditioning composite restorations prior to orthodontic bonding procedures may increase bond strength but result in cohesive failures during debonding.

3. Extension of bonding area to adjacent enamel in case of bonding to amalgam could be considered a beneficial procedure to improve bond strength of brackets but the characteristics of bond seems to be less reliable according to Weibull distribution.

\section{Acklowledgements}

The authors greatly acknowledge Cavex Holland BV, Harleem, The Netherlands for generous provision of resin composite and amalgam materials used in this study. 


\section{References}

[1] Martignon S, Ekstrand KR, Lemos MI, Lozano MP, Higuera C. Plaque, caries level, and oral hygiene habits in young patients receiving orthodontic treatment. Community Dent Health 2010;27:133-8.

[2] Sadowsky JD. An overview of treatment considerations for esthetic restorations: A review of the literature. J Prosthet Dent 2006;96:433-42.

[3] Jost-Brinkmann PG, Drost C, Can S. In-vitro study of the adhesive strengths of brackets on metals, ceramic and composite. Part 1: Bonding to precious metals and amalgam. J Orofac Orthop 1996;3:76-8.

[4] Jost-Brinkmann PG, Drost C, Can S. In-vitro study of the adhesive strengths of brackets on metals, ceramic and composite. Part 2: Bonding to porcelain and composite resin. J Orofac Orthop 1996;3:132-41. [5] Büyükyilmaz T, Zachrisson B. Improved orthodontic bonding to silver amalgam Part 2 Lathe-cut, admixed, and spherical amalgams with different intermediate resins. Angle Orthod 1998;68:337-44.

[6] Bishara SE, Ajlouni R, Oonsombat C. Bonding orthodontic bracket to composite using different surface preparations and adhesive/primers: a comparative study. World J Orthod 2003;4:343-7.

[7] Eslamian L, Ghassemi A, Amini F, Jafari A, Afrand M. Should silane coupling agents be used when bonding brackets to composite restorations? An in vitro study. Eur J Orthod 2009;31:266-70.

[8] Germeç D, Cakan U, Ozdemir FL, Arun T, Cakan M. Shear bond strength of brackets bonded to amalgam with different intermediate resins and adhesives. Eur J Orthod 2009;31:207-12.

[9] Finnema KJ, Özcan M, Post WJ, Ren Y, Dijkstra PU. In-vitro orthodontic bond strength testing: A systematic review and meta-analysis. Am J Orthod Dentofacial Orthop 2010;137:615-22.

[10] Özcan M, Alander P, Vallittu PK, Huysmans MC, Kalk W. Effect of three surface conditioning methods to improve bond strength of particulate filler resin composites. J Mater Sci Mater Med 2005;16:21-7.

[11] Özcan M. The use of chairside silica coating for different dental applications. J Prosthet Dent 2002;87:469-72.

[12] Schmage P, Nergiz I, Herrmann W, Özcan M. Influence of various surface-conditioning methods on the bond strength of metal brackets to ceramic surfaces. Am J Orthod Dentofacial Orthop 2003;123:540-6. 
[13] Matinlinna JP, Tsoi KH, de Vries J, Busscher HJ. Characterization of novel silane systems on titanium implant surfaces. Clin Oral Implants Res 2013;24:688-97.

[14] Falkensammer F, Jonke E, Bertl M, Freudenthaler J, Bantleon HP. Rebonding performance of different ceramic brackets conditioned with a new silane coupling agent. Eur J Orthod 2013;35:103-9.

[15] Matinlinna JP, Lassila LV, Özcan M, Yli-Urpo A, Vallittu PK. An introduction to silanes and their clinical applications in dentistry. Int J Prosthodont, 2004;17:155-64.

[16] Wiegand A, Attin T. Design of erosion/abrasion studies-insights and rational concepts. Caries Res 45 Supp 2011;1:53-9.

[17] Reynolds IR, Fraunhofer JA. Direct bonding in orthodontics: a comparison of attachments. Br J Orthod 1976;4:65-9.

[18] Eliades T, Brantley WA. The inappropriateness of conventional orthodontic bond strength assessment protocols. Eur J Orthod 2000;22:13-23.

[19] Bayram M, Yesilyurt C, Kusgoz A, Ulker M, Nur M. Shear bond strength of orthodontic brackets to aged resin composite surfaces: effect of surface conditioning. Eur J Orthod 2011;33:174-9.

[20] Quinn GD, Quinn JB. A practical and systematic review of Weibull statistics for reporting strengths of dental materials. Dental Mater 2010;26:135-47.

[21] Özcan M, Cura C, Valandro LF. Early bond strength of two resin cements to Y-TZP ceramic using MPS or MPS/4-META silanes. Odontology 2011;99:62-7. 


\section{Captions to tables and figures}

\section{Tables}

Table 1. The brands, chemical compositions and manufacturers of the materials used for the experiments.

Table 2. Shear bond strengths (Mean \pm standard deviation) of brackets debonded from substrates conditioned in two different ways. MPS Silane: ESPE-Sil; Universal Primer: Mononbond Plus. Different capital letters in each column and lower-case letters in each row indicate significant diferences $(p<0.05)$. *Enamel surfaces were only acid etched.

Table 3. Frequencies of failure modes in percentages. a) adhesive: when failure was between bracket and substrate with no remnants of resin on the substrate surface, b) cohesive: when the substrate failed with damaged integrity and c) mixed: when a combination of the adhesive failure from substrate and cohesive failure of the substrate or adhesive resin was present. *Dislodged before testing.

\section{Figures}

Fig. 1. Flow-chart showing experimental sequence and allocation of groups.

Fig. 2. Position of the amalgam and composite restorations and the bonded brackets on bovine teeth.

Fig. 3. Probability plot with Weibull curves $(95 \% \mathrm{Cl})$ using maximum likelihood estimation, scale and shape values for all groups. 


\begin{tabular}{|c|c|c|}
\hline Product & Chemical composition & Manufacturer \\
\hline \multirow[t]{3}{*}{ CoJet (Sand) } & $\mathrm{Al}_{2} \mathrm{O}_{3}>97 \%$ & 3M ESPE, Seefeld, Germany \\
\hline & $\mathrm{SiO}_{2}<3 \%$ & \\
\hline & $30 \mu \mathrm{m}$ particle size & \\
\hline \multirow[t]{2}{*}{ Korox (Sand) } & $\mathrm{Al}_{2} \mathrm{O}_{3}$ & Bego, Bremen, Germany \\
\hline & $50 \mu \mathrm{m}$ particle size & \\
\hline \multirow[t]{3}{*}{ ESPE-Sil } & Ethanol > 97\% & 3M ESPE, Seefeld, Germany \\
\hline & 3-Trimethoxysilyl-propyl-methacrylate $<3 \%$ & \\
\hline & Methyl ethyl ketone $<2 \%$ & \\
\hline Monobond & Ethanol $50-100 \%$ & Ivoclar-Vivadent, Schaan, \\
\hline \multirow[t]{2}{*}{ Plus } & 3-methoxysilyl-propyl-methacrylate $<2.5 \%$ & Liechtenstein \\
\hline & Methacrylated phosphoric acid ester $<2.5 \%$ & \\
\hline
\end{tabular}

Table 1. The brands, chemical compositions and manufacturers of the materials used for the experiments. 


\begin{tabular}{lcc}
\hline Bonding Substrate & \multicolumn{2}{c}{ Surface Conditioning } \\
\hline & SiO2 + MPS Silane & Al2O3 + Universal Primer \\
\cline { 2 - 3 } Amalgam & $10.96 \pm 1.16^{\mathrm{A}, \mathrm{a}}$ & $12.41 \pm 1.53^{\mathrm{A}, \mathrm{a}}$ \\
Enamel + Amalgam & $16.29 \pm 3.16^{\mathrm{A}, \mathrm{a}}$ & $16.36 \pm 8.31^{\mathrm{A}, \mathrm{a}}$ \\
Enamel + Composite & $25.89 \pm 6.62^{\mathrm{B}, \mathrm{a}}$ & $20.64 \pm 3.69^{\mathrm{B}, \mathrm{a}}$ \\
Composite $^{3}$ & \multicolumn{2}{c}{$26.72 \pm 4.03^{\mathrm{B}}$} \\
Enamel $^{*}$ & $31.37 \pm 2.85^{\mathrm{B}, \mathrm{a}}$ \\
\hline
\end{tabular}

\begin{tabular}{|l|c|c|}
\hline Substrate & \multicolumn{2}{|c|}{ Surface Conditioning } \\
\hline & $\mathbf{S i O}_{2}+$ MPS Silane & $\mathbf{A l}_{2} \mathbf{O}_{3}+$ Universal Primer \\
\hline Amalgam & $10.96 \pm 1.16^{\mathrm{A}, \mathrm{a}}$ & $12.41 \pm 1.53^{\mathrm{A}, \mathrm{a}}$ \\
\hline Enamel-Amalgam & $16.29 \pm 3.16^{\mathrm{A}, \mathrm{a}}$ & $16.36 \pm 8.31^{\mathrm{A}, \mathrm{a}}$ \\
\hline Enamel-Composite & $25.89 \pm 6.62^{\mathrm{B}, \mathrm{bc}}$ & $20.64 \pm 3.69^{\mathrm{B}, \mathrm{b}}$ \\
\hline Composite $^{\mid 2} 26.72 \pm 4.03^{\mathrm{B}, \mathrm{c}}$ \\
\hline Enamel $^{*}$ & $31.37 \pm 2.85^{\mathrm{B}, \mathrm{c}}$ & $29.97 \pm 3.31^{\mathrm{B}, \mathrm{c}}$ \\
\hline
\end{tabular}

Table 2. Shear bond strengths (Mean \pm standard deviation) of brackets debonded from substrates conditioned in two different ways. MPS Silane: ESPE-Sil; Universal Primer: Mononbond Plus. Different capital letters in each column and lower-case letters in each row indicate significant diferences $(p<0.05)$. ${ }^{* *}$ Enamel surfaces were only acid etched. 


\begin{tabular}{|c|c|c|c|c|}
\hline & \multicolumn{4}{|c|}{ Failure Modes (\%) } \\
\hline & Dislodged* & Adhesive & Mixed & Cohesive \\
\hline Enamel-Composite $\left(\mathrm{SiO}_{2}\right)$ & 0 & 10 & 60 & 30 \\
\hline Enamel-Amalgam $\left(\mathrm{SiO}_{2}\right)$ & 0 & 80 & 20 & 0 \\
\hline Enamel & 0 & 20 & 80 & 0 \\
\hline Composite $\left(\mathrm{SiO}_{2}\right)$ & 0 & 0 & 30 & 70 \\
\hline Amalgam $\left(\mathrm{SiO}_{2}\right)$ & 0 & 90 & 10 & 0 \\
\hline Composite $\left(\mathrm{Al}_{2} \mathrm{O}_{3}\right)$ & 1 & 10 & 20 & 70 \\
\hline Amalgam $\left(\mathrm{Al}_{2} \mathrm{O}_{3}\right)$ & 1 & 100 & 0 & 0 \\
\hline Enamel-Amalgam $\left(\mathrm{Al}_{2} \mathrm{O}_{3}\right)$ & 0 & 90 & 10 & 0 \\
\hline $\begin{array}{l}\text { Enamel-Composite } \\
\left(\mathrm{Al}_{2} \mathrm{O}_{3}\right)\end{array}$ & 0 & 10 & 70 & 20 \\
\hline
\end{tabular}

Table 3. Frequencies of failure modes in percentages. a) adhesive: when failure was between bracket and substrate with no remnants of resin on the substrate surface, b) cohesive: when the substrate failed with damaged integrity and c) mixed: when a combination of the adhesive failure from substrate and cohesive failure of the substrate or adhesive resin was present. *Dislodged before testing. 


\section{Figures:}

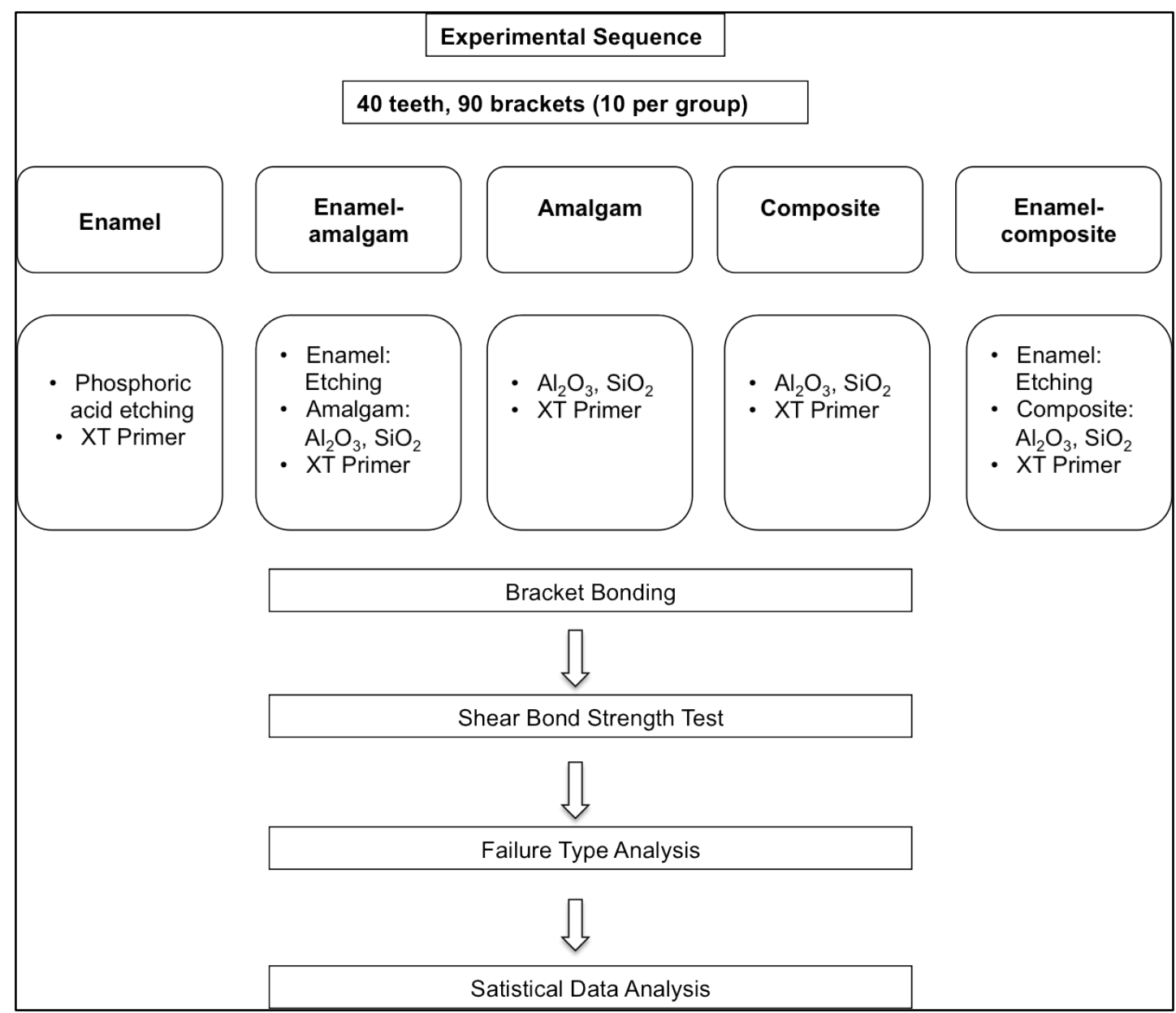

Fig. 1. Flow-chart showing experimental sequence and allocation of groups. 


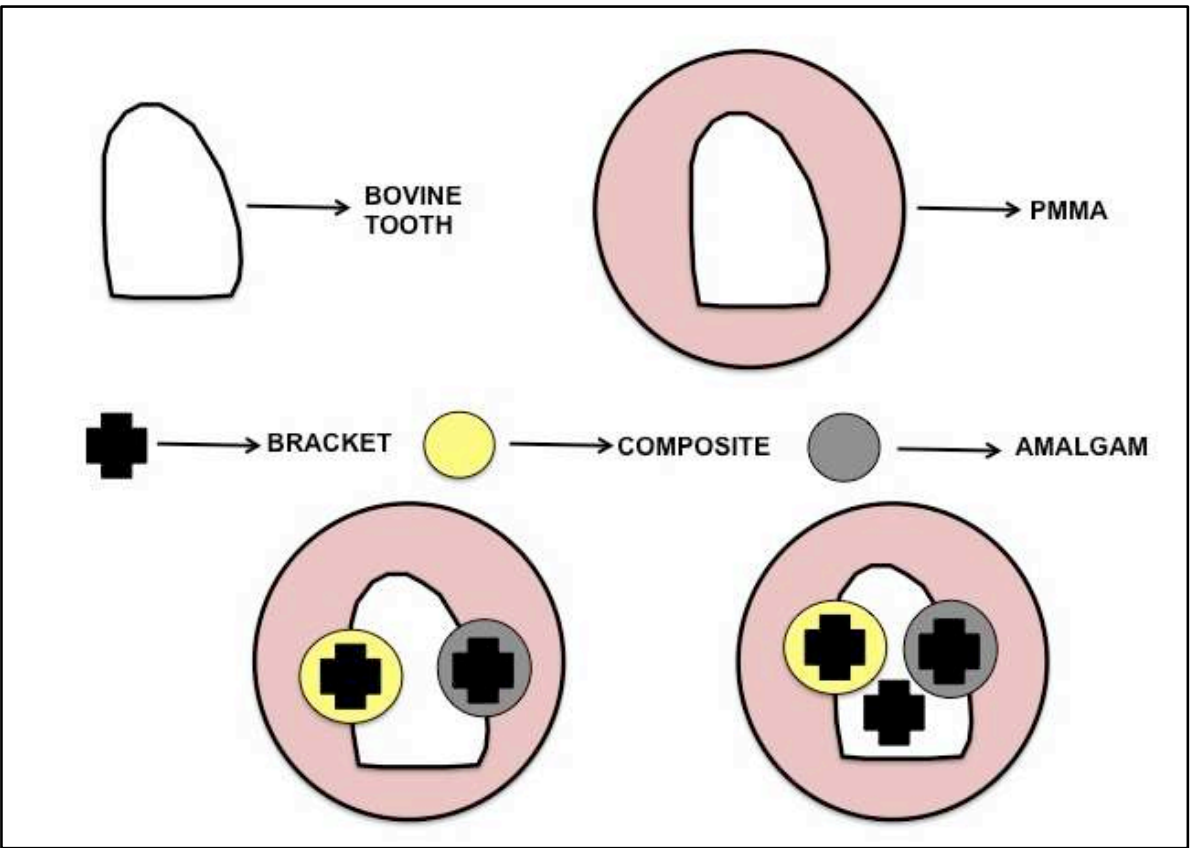

Fig. 2. Position of the amalgam and composite restorations and the bonded brackets on bovine teeth.

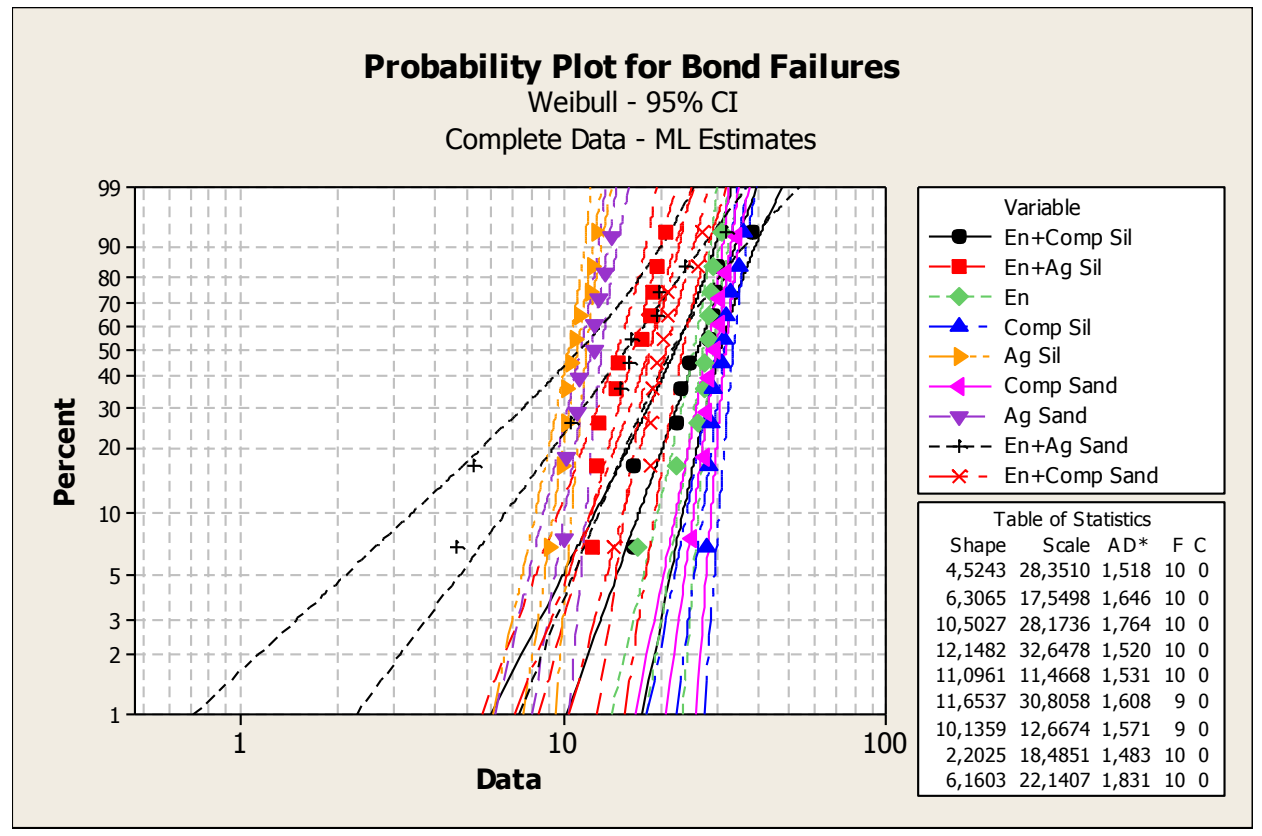

Fig. 2. Probability plot with Weibull curves $(95 \% \mathrm{Cl})$ using maximum likelihood estimation, scale and shape values for all groups. 